\title{
Measurements of Soft-Tissue Mechanical Properties to Support Development of a Physically Based Virtual Animal Model
}

\author{
Cynthia Bruyns ${ }^{1,3}$ and Mark Ottensmeyer ${ }^{2}$ \\ 1 BioVis Technology Center, NASA Ames Research Center \\ cbruyns@mail.arc.nasa.gov \\ 2 Simulation Group, CIMIT \\ mpo@alumni.mit.edu \\ 3 National Biocomputation Center, Stanford University \\ bruyns@biocomp.stanford.edu
}

\begin{abstract}
Though the course of ongoing development of a dynamic "Virtual Rat", the need for physically based simulation has been established. To support such physical modeling, data regarding the material properties of various tissues is required. We present the results of in vitro testing of rat organ tissues and discuss preliminary comparisons of the results with a Finite Element simulation of the test scenario and a method to extract parameters from test data.
\end{abstract}

\section{Introduction}

The goals of the Virtual Rat Project are to reduce the need for training on animals, create a system for training that allows for remote collaboration, and provide a system for training that is rich in content and flexible in user interaction.

The previous development of the system has focused on imaging and reconstruction of the anatomy of the Virtual Rat and development of the surgical manipulations commonly performed in an animal dissection.

The initial modeling efforts have created a combined surface and volumetric representation of the rat anatomy. These 3D models are simulated within a physically based modeling framework to allow the user to probe, cut and remove the various tissues [8],4]. Within this framework, each organ is represented as surface and/or volumetric meshes and assigned dynamic properties that allow the tissue to respond to user interaction.

During the development of the simulation system, the need for more realistic soft-tissue deformations has been identified. Currently, we are using a mass-spring modeling paradigm. Therefore, surface representations of tissues deformations are commonly represented as bending of the surface. When a user interacts with the tissue locally, without "high spring constants," the deformations can look unrealistic. For volumetric objects however, interactions with the tissues become less localized and when coupled with volume preserving methods, can provide "more appealing" deformations. 
However, it is more desirable to have the behavior of the object depend on the constitutive properties of the object being simulated and less dependent on the geometry used to represent it. Moreover, we want to avoid manipulating such low-level parameters such as spring constants and move toward modeling the organ as a continuous medium.

There are some issues, however to consider, if one wants to build a surgical simulation trainer that will provide the user with a meaningful learning experience. For example, the behavior of these tissues with which the user is learning to interact must be accurately recreated. Otherwise one runs the risk of training the user to perform a given task incorrectly.

Using a mass-spring model, there is very little real-world material parameter correlation. The only parameters that control the amount of deformation of the simulated soft tissue are the spring stiffness and damping coefficients. These parameters are often approximated for visual quality.

Alternative soft-tissue modeling paradigms, such as Finite Elements can use material parameters such as Poisson's ratio and Elastic Modulus directly in their computations. It is these properties along with geometry and loading and boundary conditions that can predict how a tissue will respond to a given interaction.

Mass-spring models however, have the advantage of being very simple to solve and are an order of magnitude faster than the more rigorous Finite Element Models [12]. In addition to computational efficiency, mass-spring systems have the benefit of allowing topological modifications to be made interactively.

Our goal is to combine the benefits of both modeling approaches to arrive at a simulation that is both fast and precise in its representation of tissue deformation.

Others have noted the need for incorporating material parameters into surgical simulations [7]. Moreover, others have expressed the need for a database of these parameters that can be easily accessed and shared throughout the surgical simulation community [5]. We aim to create a baseline protocol for obtaining soft-tissue properties and using this protocol we are beginning to develop an integrated anatomical atlas of baseline material properties for the Virtual Rat for use in the training system, and so that the effects of weightlessness, disease, and other abnormalities can be compared.

\section{Methods}

\subsection{Necropsy}

The testing protocol was approved by the NASA Ames Research Center IACUC Committee under Protocol Number 01-033-2. On separate occasions, four female rats (Rattus norvegicus) of approximately $250 \mathrm{~g}$ were euthanized through inhalation of CO as accepted by the AVMA. For the in situ testing, the abdominal cavity was opened immediately after inhalalation and the instrument was placed over the tested organ. In this manner, blood was still inside the organ and the surrounding organs supported the tested organ, much as they would in vivo. One note however, is that the opening of the body cavity and rupture of 
the diaphragm did change the boundary conditions of the organ from exact in vivo conditions, but every effort was made to keep the variations to a minimum. For the in vitro testing the organs were removed and placed on a hard glass surface. The organs were kept moist and the time that had past post-mortem while testing the organs was noted so that the variation of material properties with time after death could be investigated.

\subsection{TeMPeST 1-D Testing}

To acquire tissue property data covering the range of frequencies relevant to simulation with haptic feedback, the TeMPeST 1-D instrument [9], 10] was used to deform the tissues and record the force/displacement response. The TeMPeST 1-D makes use of a voice coil motor to drive a right-circular punch with a $5 \mathrm{~mm}$ diameter, normal to the tissue over a range of motion of $\pm 500 \mu \mathrm{m}$ with a force up to $300 \mathrm{mN}$. The open loop bandwidth of the instrument is approximately $100 \mathrm{~Hz}$, and within these limits, an arbitrary trajectory can be generated. Absolute force and relative position are sampled at frequencies up to $2 \mathrm{kHz}$.

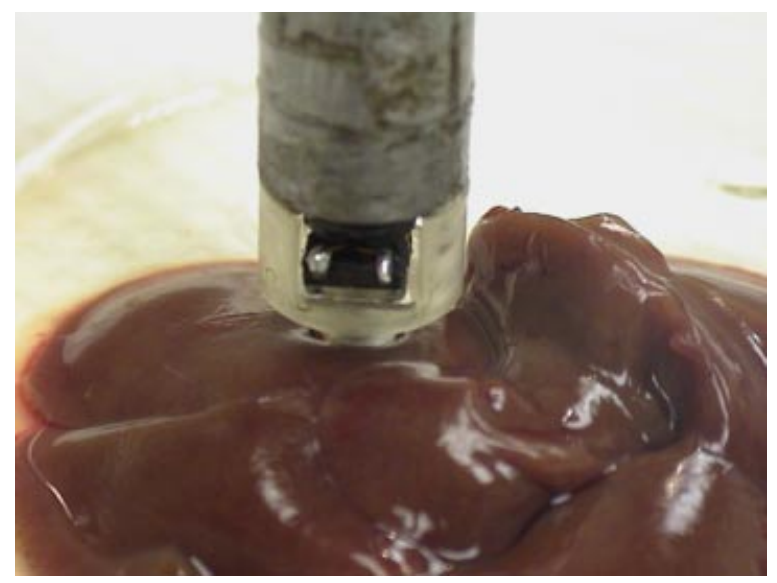

Fig. 1. In vitro testing of rat liver with TeMPeST 1-D instrument

A series of tests on the heart, liver, kidney and abdominal muscle layer were conducted with the organs/tissues in situ and in vitro; Figure11demonstrates the test apparatus. Previous use of the instrument includes testing on porcine solid organ tissue in vivo. Live testing complicates the boundary conditions of the tissue relative to in vitro testing, and more importantly, introduces disturbances in the position of the tissue due to either cardiac or pulmonary motion. Cardiac motion could not be suspended (for obvious reasons), but ventilation of the pig could be halted while data was acquired. Since the rat testing was conducted on sacrificed animals, longer duration sampling could be conducted, and the motion disturbances were not present. Table 1 summarizes the tests conducted (see also 
[3]), however in this paper we focus on the data acquired from the latter two rats. As a result of a more restricted set of tissues and an improved measurement protocol, these tests were more informative and reliable.

Table 1. Summary of organ testing, including mode of testing, time post mortem and type of excitation.

\begin{tabular}{|c|c|c|c|c|}
\hline rat ID \# & organ/tissue & tests & time & test excitation \\
\hline 1 & $\begin{array}{l}\text { heart (3 tests) } \\
\text { liver }(4) \\
\text { abdominal muscle (1) } \\
\text { kidney (4) } \\
\text { liver }(3)\end{array}$ & $\begin{array}{c}\text { in } \text { situ } \\
\quad " \\
\quad " \\
\text { in vitro } \\
\quad "\end{array}$ & $\begin{array}{c}4-7 \text { min. } \\
11-21 \\
25 \\
35-51 \\
57-62\end{array}$ & $\begin{array}{c}\text { linear chirp } \\
" \\
" \\
\text { lin. \& exponential chirp } \\
\text { exp. chirp }\end{array}$ \\
\hline 2 & $\begin{array}{l}\text { lung (5) } \\
\text { liver (8) } \\
\text { kidney (6) }\end{array}$ & $\mid \begin{array}{c}\text { in } \\
\text { vitro } \\
" \\
"\end{array}$ & $\begin{array}{c}6-25 \\
29-52 \\
59-76\end{array}$ & $\begin{array}{c}\text { exp. chirp \& sinusoidal } \\
\text { exp. chirp } \\
"\end{array}$ \\
\hline 3 & $\begin{array}{l}\text { kidney (r\&l) (18) } \\
\text { liver (10) } \\
\text { spleen (3) }\end{array}$ & $\mid \begin{array}{c}\text { in } \text { vitro } \\
" \\
"\end{array}$ & $\begin{array}{c}0-52 \\
56-79 \\
86-95\end{array}$ & $\begin{array}{c}\text { exp. chirp } \\
", \\
"\end{array}$ \\
\hline 4 & $\begin{array}{l}\text { kidney (r\&l) (18) } \\
\text { liver (9) }\end{array}$ & $\begin{array}{c}\text { in vitro } \\
"\end{array}$ & $\begin{array}{c}0-54 \\
57-78\end{array}$ & $\begin{array}{c}\text { exp. chirp } \\
\text { " }\end{array}$ \\
\hline
\end{tabular}

\subsection{Finite Element Analysis}

The imaging and 3D reconstruction of the Virtual Rat was performed as described in 2]. Figure 22 illustrates the high-resolution MDCT images and the resulting $3 \mathrm{D}$ reconstruction of the kidneys.
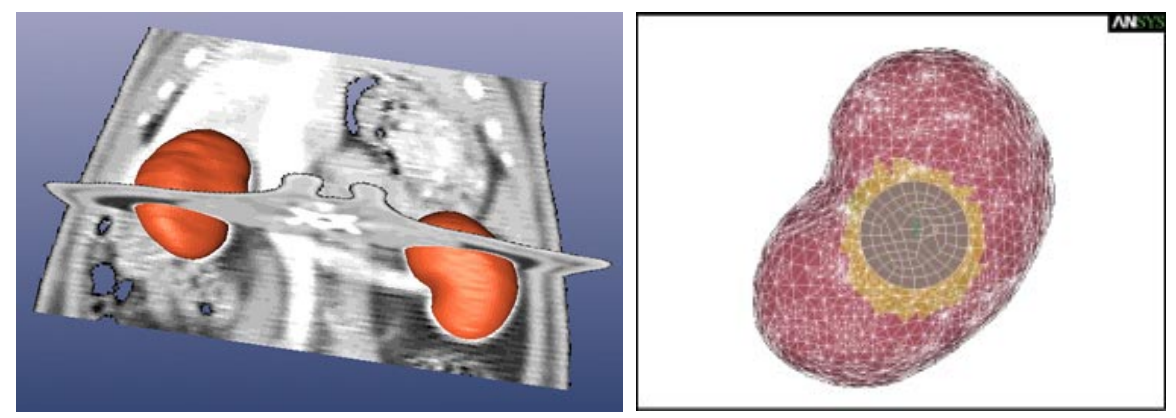

Fig. 2. MDCT imaging (left) and reconstructed FEM kidney with indenter contact zone defined (right)

For comparison with the actual testing, a reconstructed kidney was imported into TrueGrid (XYZ Scientific Applications, Inc, Livermore, Ca.) solid modeling 
software, and modified to obtain the Finite Element model of the organs. This software allows models obtained from common 3D reconstruction software, such as those obtained from General Marching Cubes algorithms to be converted to hexahedral finite elements. Then using the ANSYS (ANSYS Corporation, Cannonsburg, Pa.) Finite Element Analysis software, the test scenario was simulated using 95-node non-linear brick elements for the tissue and 45-node linear brick elements for the indenter tip. Contact elements were also placed between the tissue and indenter surface to simulate any possible loss of contact between the indenter and the tissue surface during testing. A pilot node was used to specify the position of the indenter as recorded during testing. Nodes were selected at the base of the tissue and fixed with respect to position, simulating their contact with the petri dish. Figure 2 demonstrates the initial triangulated kidney surface and the final solid model using the TrueGrid software.

Using this Finite Element model, an initial estimate for Young's Modulus was made and the corresponding force that resulted from the indenter-tissue contact was examined, and the initial guess was updated accordingly.

\section{Results}

\subsection{Testing}

Figures 3(a) and (b) show the mean and standard deviations of the ratios of the Fourier transforms of the position and force (i.e. non-parametric representation of compliance transfer function) for the kidney and liver (four and two organs, respectively, and 11-13 data sets for each of three loading conditions).

Additional tests on more different organs will be necessary to establish reliable mean and deviation estimates for the tissues. One result that can be immediately seen is that over the range of frequencies examined, the tissues have a small phase lag and a decrease in the magnitude of compliance. While this would indicate some viscous component, it is not as severe as a first order spring-dashpot model, so as a first approximation, the material could be treated as elastic. This result immediately simplifies the job of the simulation programmer, since time dependent effects can be omitted from the model. A more complex model could include non-linear elasticity, inhomogeneity and anisotropy, as well as terms to describe the frequency dependent properties. To date, insufficient data has been obtained to determine the post-mortem changes in material properties.

\subsection{Implementation into a Mass-Spring System}

[12] points out that assigning the same stiffness to all springs within a virtual organ fails to simulate a uniform elastic membrane for equilibrium calculations. Instead he proposes a derivation of stiffness that varies as triangle area over edge length squared. Using this derivation and the 2D Young's Modulus for surface-based representations and the 3D Young's Modulus for volumetric representations, we begin to see the effect that the choice of stiffness parameters has 
(a)
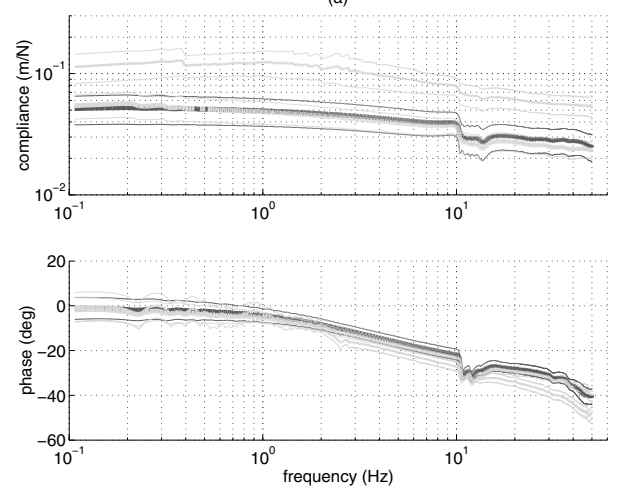

(b)
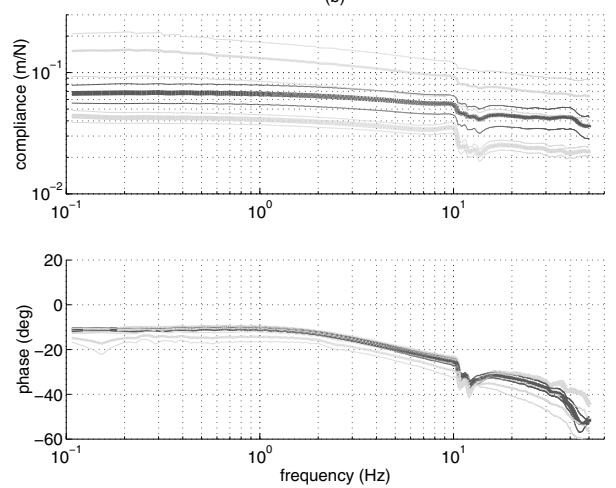

Fig. 3. Compliance of kidney (a) and liver (b) in vitro vs. frequency, mean \pm standard deviation for all in vitro measurements. Heavy lines indicate high preload force (42 vs. $21 \mathrm{mN})$, dark colored lines indicate high amplitude (60 vs. $30 \mathrm{mN})$
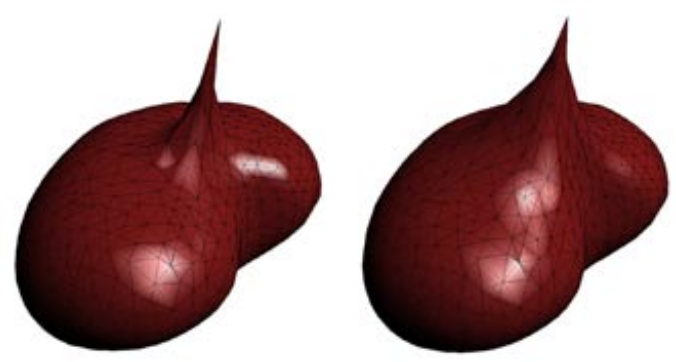

Fig. 4. (a) Uniform spring constant (b)Derived from material properties

on the simulation of these objects using springs. Figure 4 shows the effect that the choice of spring constants has on the static deformation on the simulated kidney 1

\section{Discussion and Future Work}

The figures above demonstrate the difference that the choice of material parameters has on the simulation of soft-tissues. As expected, mass-spring systems with estimated material parameters alone, does not go far enough to try to capture realistic tissue behavior. Instead by using the techniques described, one can approach the combination of the realism of finite element simulations with the speed and simplicity of mass-spring systems. As pointed out by [12] mass-spring

\footnotetext{
${ }^{1}$ Inertial effects due to distribution of mass throughout the object does not affect the static deformation. However, prior to beginning the simulation mass is redistributed in the object as a function of adjacent Voronoi area/volume for each node [4].
} 
simulations cannot exactly simulate the stresses within a material under all stress conditions, however using physically derived stiffness constants, is a first attempt to incorporate the constitutive material of the object being simulated in such a modeling paradigm.

Although the imaging was performed on a different animal than was tested, we were careful to match the age and the size of the animals for a realistic representation of the kidney geometry. However, because the tissues were extracted, we were not able to recreate the loading scenario of the liver. In future experiments we will image the ex vivo tissues during loading to provide better approximations for the organ geometry and boundary conditions.

However, given the available results, certain notable features of the measured kidney compliance are apparent that will need to be reproduced in the finite element modeling. For example, with higher preloads, the contact region between the kidney and the dish is expected to be larger, and simultaneously, assuming a hyperelastic characteristic in the tissue itself, one would expect to, and does observe a lower compliance (higher stiffness) for this condition. At the same time, variations in amplitude of vibration (of those examined) do not result in significant changes in stiffness. The liver results are not as clear, most likely because the liver geometry is less constrained. Further tests with imaging will likely clarify the results, and permit the extraction of material properties.

In addition, although the Virtual Rat is currently being developed as a dissection trainer, one can foresee several uses for the simulation system including animal surgery and care. Therefore we are also planning on obtaining the material parameters of the same tissues in vivo.

We also plan to investigate how, by using other testing techniques, we can find the yield strength of these tissues. Currently the yield strength is approximated for visual realism during the simulation of manipulations such as cutting. However, it is desirable to be able to determine the point of plastic deformation and eventual fracture of the surface during puncture [11].

\section{Acknowledgements}

This work was supported by grants to the NASA Ames BioVis Technology Center and the National Biocomputation Center. Special thanks to Anil Menon, Marilyn Vasques, Kim Winges and Richard Boyle. Special thanks also to Robert Rainsberger and Matthew Koebbe of XYZ Scientific Applications, Inc. This work was also aided by discussions with Mark Rodamaker from ANSYS, Corporation. Portions of this work were also supported by the Department of the Army, under contract number DAMD17-99-2-9001. The views and opinions expressed do not necessary reflect the position or the policy of the government, and no official endorsement should be inferred. 


\section{References}

1. Brown, J., Sorkin, S., Bruyns, C., Latombe, JC., Montgomery, K., Stephanides, M.: Real-time Simulation of Deformable Objects: Tools and Application, Computer Animation 2001, Seoul, Korea,(6-8 November 2001).

2. Bruyns, C; Montgomery, K; Wildermuth, S.: Advanced Astronaut Training/Simulation System for Rat Dissection, Medicine Meets Virtual Reality 02/10, J.D. Westwood, et al. (Eds.), Newport Beach, CA. IOS Press. (24-27 Jan 2001) 75-81. 2001.

3. Bruyns, C., Ottensmeyer, M.P.: The Development of a Physically Based Virtual Animal Model using Soft-Tissue Parameter Testing. 5th IASTED International Conference on Computer Graphics and Imaging, Kauai, HI, (12-14 Aug 2002) Accepted for publication. 2002.

4. Bruyns, C., Senger, S., Menon, A., Montgomery, K., Wildermuth, S., Boyle, R.: A survey of interactive mesh cutting techniques and a new method for implementing generalized interactive mesh cutting using virtual tools. The Journal of Visualization and Computer Animation. (In Press)

5. Open discussion at Common Anatomical Modeling Language Round Table, Utrecht, Netherlands, October 2001.

6. Fung, Y.C.: Biomechanics: Mechanical Properties of Living Tissues 2nd Edition. Springer Verlag, NT. 1993

7. Miller K., Chinzei K., Orssengo G. and Bednarz P.: Mechanical properties of brain tissue in-vivo: experiment and computer simulation. J. Biomechanics, Vol. 33, pp. 1369-1376, 2000.

8. Montgomery, K., Bruyns, C., Brown, J., Sorkin, S., Mazzella, F., Thonier, G., Tellier, A., Lerman, B., Menon A.: Spring: A General Framework for Collaborative, Real-time Surgical Simulation. Proceedings of Medicine Meets Virtual Reality 02/10, J.D. Westwood, et al. (Eds.), Newport Beach, CA. IOS Press. (23-26 Jan 2002) 296-303.

9. Ottensmeyer, M.P., Salisbury, J.K. Jr.: In Vivo Data Acquisition Instrument For Solid Organ Mechanical Property Measurement, Proceedings of the Medical Image Computing and Computer-Assisted Intervention - MICCAI'01. 4th International Conference, Utrecht, The Netherlands (14-17 Oct 2001) 975-982

10. Ottensmeyer, Mark P.: In vivo measurement of solid organ visco-elastic properties, Proceedings of Medicine Meets Virtual Reality 02/10, J.D. Westwood, et al. (Eds.), Newport Beach, CA. IOS Press. (23-26 Jan 2002) 328-333.

11. Terzopolous, D., Fleicher, K.: Modeling inelastic deformation: viscoelasticity, plasticity, fracture. Computer Graphics Proceedings of SIGGRAPH 1988; 269-278.

12. van Gelder, A.: Approximate simulation of elastic membranes by triangulated spring meshes. Journal of Graphics Tools, 3(2): 21-41, 1998. 